\title{
Unilateral massive hydrothorax in a gynecologic patient with pseudo-Meigs' syndrome -A case report-
}

\author{
Tae Soo Hahm, Ji Sun Ham, and Ji Yeong Kang \\ Department of Anesthesiology and Pain Medicine, Samsung Medical Center, Sungkyunkwan University School of Medicine, Seoul, \\ Korea
}

Pseudo-Meigs' syndrome is characterized by the presence of a benign ovarian tumor associated with ascites and a right-sided hydrothorax. The major problem associated with pseudo-Meigs' syndrome is the respiratory distress caused by a giant mass in the peritoneal space, massive ascites and pleural effusion. Even if there are no respiratory problems prior to surgery, potential respiratory dysfunction can occur during the peri-anesthetic period, leading to hypoxia, hypercapnea and respiratory acidosis. We report a case of hypoxia during recovery from anesthesia in a gynecological patient with pseudo-Meigs' syndrome. (Korean J Anesthesiol 2010; 58: 202-206)

Key Words: Hydrothorax, Hypoxia, Pseudo-Meigs' syndrome.

Pseudo-Meigs' syndrome is characterized by the presence of a benign ovarian tumor associated with ascites and a rightsided hydrothorax. Fibroma cases of benign ovarian tumors are called Meigs' syndrome, and the other cases of ovarian cancer are defined as pseudo-Meigs' syndrome[1,2].

These syndromes can produce respiratory distress caused by massive ascites and pleureal effusion, which can lead to respiratory dysfunction, such as hypoxia, hypercapnea and respiratory acidosis. Delicate anesthetic management is needed during pre- and post-operation because hydrothorax and acites are produced rapidly.

We report a pseudo-Meigs' syndrome patient who experienced a respiratory dysfunction with hypoxia caused by a right-sided hydrothorax during emergence from general anesthesia.

\section{Case Report}

A female patient, 46 years old and $70 \mathrm{~kg}$ of body weight, was admitted to hospital with a palpable abdominal mass as the chief complaint that started a few months earlier. Vaginal sonography showed a cystic tumor $(10.2 \times 6.8 \mathrm{~cm})$ in the right ovary. Abdominal CT revealed multicysts suggesting a borderline epithelial tumor or a benign tumor. No significantly enlarged lymph node was found. Massive ascites in the peritoneal space and small amount of hydrothorax in the right side were observed.

Her prior medical history was hypertension, which was well controlled by medication. No other medical conditions were present. The laboratory blood tests prior to surgery were within the normal limits, and the serum albumin level was also normal

Received: August 26, 2009. Revised: 1st, September 8, 2009; 2nd, September 18, 2009. Accepted: October 1, 2009.

Corresponding author: Tae Soo Hahm, M.D., Department of Anesthesiology and Pain Medicine, Samsung Medical Center, Sungkyunkwan University School of Medicine, 50, Ilwon-dong, Gangnam-gu, Seoul 135-230, Korea. Tel: 82-2-3410-0364, Fax: 82-2-3410-0361, E-mail: tshahm@skku.edu (C)This is an open-access article distributed under the terms of the Creative Commons Attribution Non-Commercial License (http:// creativecommons.org/licenses/by-nc/3.0/), which permits unrestricted non-commercial use, distribution, and reproduction in any medium, provided the original work is properly cited. 
( $4 \mathrm{mg} / \mathrm{dl}$ ). Electrocardiography was also nonspecific. The chest $\mathrm{X}$-ray taken one week before surgery showed a small amount of a right-sided hydrothorax that was expressed as a costophrenic angle blunting image. No other findings were found (Fig. 1). There were no preoperative symptoms, such as dyspnea or chest pain.

Premedication in anesthesia was not performed, and the vital signs upon the arrival at the operation room were a blood pressure, pulse rate and $\mathrm{SpO}_{2}$ of $150 / 70 \mathrm{mmHg}$, 90/ min and $100 \%$ in room air, respectively. General anesthesia was induced by total intravenous anesthesia (TIVA) with propofol and remifentanil, and rocuronium $0.7 \mathrm{mg} / \mathrm{kg}$ was then injected after unconsciousness. Subsequently, an endotracheal tube was intubated, and a bilateral clear lung sound was ausculatated. The depth of the tube was set to $21 \mathrm{~cm}$. Anesthesia was maintained with $\mathrm{O}_{2} 1 \mathrm{~L} / \mathrm{min}$, air $1 \mathrm{~L} / \mathrm{min}$, propofol, and remifentanil, and mechanical ventilation was set to a tidal volume and respiratory rate of $450 \mathrm{ml}$ and $8 / \mathrm{min}$, respectively, to maintain a peak inspiratory pressure (PIP) and end-tidal $\mathrm{CO}_{2}$ concentration $\left(\mathrm{Et}^{\mathrm{CO}_{2}}\right.$ ) of $17 \mathrm{cmH}_{2} \mathrm{O}$ and $30 \mathrm{mmHg}$, respectively.

The patient was placed in the supine position. The left radial artery was catheterized to monitor the continuous blood pressure. The arterial blood gas analysis (ABGA) taken before the surgical incision showed a $\mathrm{PaO}_{2}$ of $163 \mathrm{mmHg}$ in a $\mathrm{FiO}_{2}$ of 0.6. There was bilateral clear lung sound and no tracheal secretion during endotracheal suction. Five $\mathrm{cmH}_{2} \mathrm{O}$ of positive endexpiratory pressure (PEEP) was applied to prevent atelectasis by increasing the functional residual volume.

The vital signs during surgery were stable, and a $\mathrm{SpO}_{2}$ of $100 \%$ was maintained. Approximately $500 \mathrm{ml}$ of ascites were drained after the peritoneal opening, and there were no specific

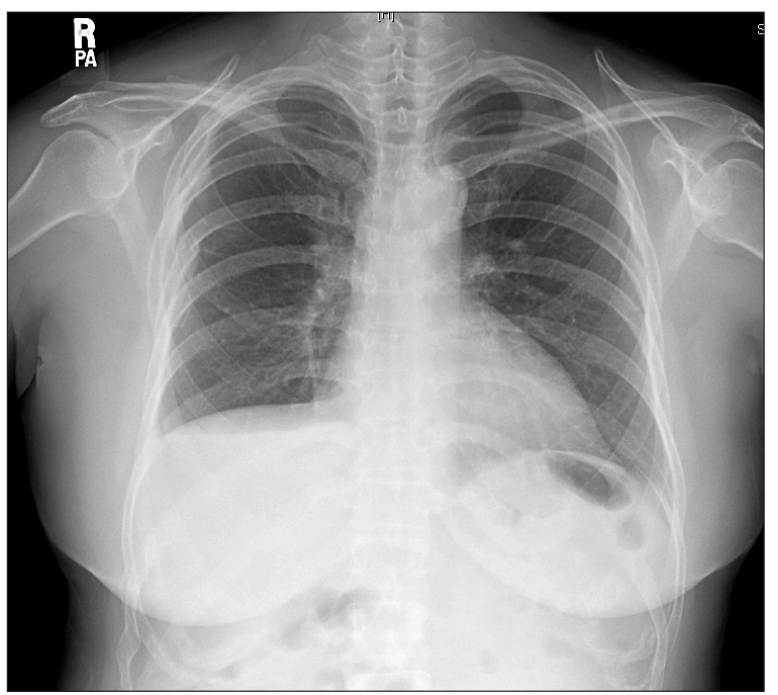

Fig. 1. Chest radiograph 1 week before surgery shows blunting of the right costopleural angle. changes in her vital signs or $\mathrm{EtCO}_{2}$. A total right salphingooophorectomy with a hysterectomy was performed for 3 hours successfully. The pathological result of the tumor was a $25 \times 18$ $\times 8 \mathrm{~cm}$ sized ovarian mucinous cystadenoma. Ringer's lactate $(1,300 \mathrm{ml})$ and pentastarch (pentaspan ${ }^{\circledR}$, Je-il pharmacy, Korea) $(500 \mathrm{ml})$ were administered intravenously. The urine output and estimated blood loss was $150 \mathrm{ml}$ and $200 \mathrm{ml}$, respectively.

Glycopyrrolate $0.4 \mathrm{mg}$ and pyridostigmine $15 \mathrm{mg}$ were injected when the patient began to restore spontaneous breathing after surgery. After 10 minutes, the tidal volume had increased to 70-100 ml. However, the $\mathrm{SpO}_{2}$ decreased abruptly to $65 \%$, and a decrease in tidal volume $(<30 \mathrm{ml})$ was also observed. Positive pressure ventilation was performed, and $\mathrm{SpO}_{2}$ increased to 100 $\%$ within 1 minute, but the ABGA results at this time showed the following: $\mathrm{PaO}_{2} 58 \mathrm{mmHg}, \mathrm{PaCO}_{2} 46 \mathrm{mmHg}$, and $\mathrm{pH} 7.35$. This condition was confirmed as respiratory acidosis caused by hypoxia and hypercapnea. Therefore, mechanical ventilation with $5-10 \mathrm{cmH}_{2} \mathrm{O}$ of PEEP was applied with midazolam $5 \mathrm{mg}$ and vecuronium $4 \mathrm{mg}$. The following parameters were used: $\mathrm{FiO}_{2}$ 1.0, a tidal volume of $400 \mathrm{ml}$, and a respiratory rate of 8/ min. Because there was a decreased breathing sound on the right lung field, and the chest X-ray taken one week before surgery showed a small amount of hydrothorax, portable chest radiography was called, which revealed a total haziness on the right hemithorax (Fig. 2). A large amount of right sided pleural effusion was considered. To solve this problem, lasix $10 \mathrm{mg}$ was injected, and the patient was moved to the post anesthesia care unit (PACU) so a chest tube could be inserted.

In the PACU, mechanical ventilation set to the following: $\mathrm{FiO}_{2} 0.54$, tidal volume $400 \mathrm{ml}$, and respiratory rate $8 / \mathrm{min}$. The internal medicine physician on call inserted the chest tube, and

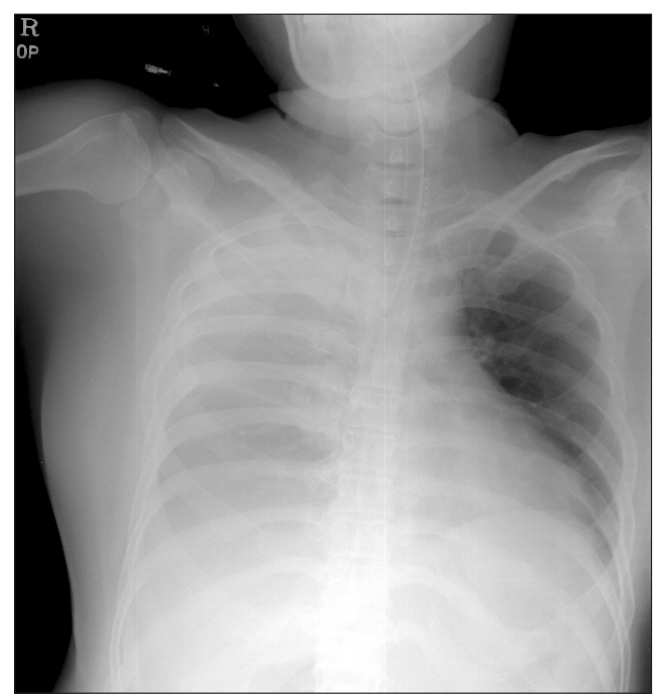

Fig. 2. Chest radiograph immediately after surgery shows a massive right-sided pleural effusion. 


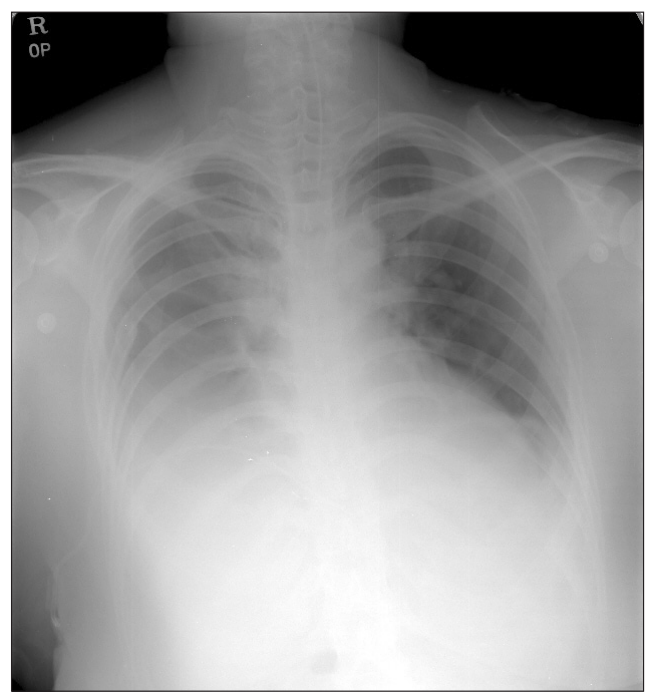

Fig. 3. Chest radiograph shows the chest tube inserted after the right thoracostomy.

a $750 \mathrm{ml}$ pleural effusion was drained. After 30 minutes, a chest radiography was performed to confirm the position of the chest tube (Fig. 3). The pathophysiological test of a hydrothorax was later shown to be devoid of malignant cells. One and half hours after inserting the chest tube and the patient became alert, and the following ABGA was normalized: $\mathrm{PaO}_{2} 190 \mathrm{mmHg}, \mathrm{PaCO}_{2}$ $43 \mathrm{mmHg}$, and $\mathrm{pH}$ 7.37. The condition of the patient was stable with a blood pressure and pulse rate of 120/60 $\mathrm{mmHg}$ and 70/ min, respectively, so she was extubated. $\mathrm{O}_{2} 6 \mathrm{~L} / \mathrm{min}$ via a facial mask with a reservoir bag was applied to maintain the $\mathrm{SpO}_{2}$ at $100 \%$. After one hour, the ABGA was as follows: $\mathrm{PaO}_{2} 212$ $\mathrm{mmHg}, \mathrm{PaCO}_{2} 38 \mathrm{mmHg}$, and $\mathrm{pH}$ 7.37. The vital signs were also stable, so the patient was transferred to the gynecologic ward.

The chest tube was removed on the $2^{\text {nd }}$ day because there was no more drainage, and a $\mathrm{SpO}_{2} 98 \%$ was maintained without an $\mathrm{O}_{2}$ supply. The chest radiograph revealed a bilateral clear lung field (Fig. 4). She was finally discharged on the 6th day after surgery without symptoms and abnormal signs.

\section{Discussion}

In gynecological diseases, Meigs' and pseudo-Meigs' syndromes, which are characterized by a benign ovarian tumor, ascites and hydrothorax, can cause severe complications, such as respiratory distress from the massive tumor and large amounts of ascites and hydrothorax during anesthesia [3]. These two syndromes respond in precisely the same manner in both the clinical manifestation and treatment, and even the ascites and hydrothorax are well resolved after resecting the tumor. This case was a pathophysiologically benign ovarian tumor of a

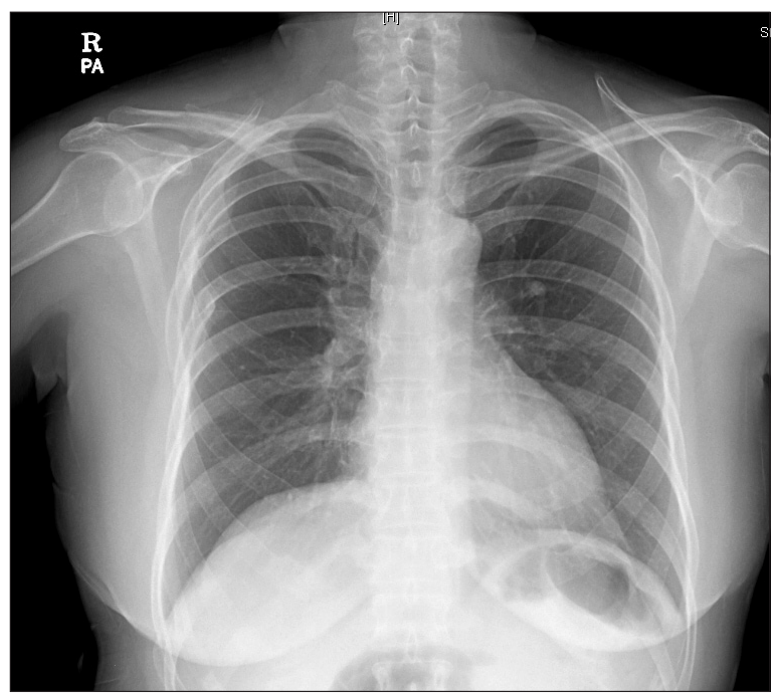

Fig. 4. The chest tube was removed 2 days after the thoracostomy. The chest radiograph shows normal findings.

mucinous cystadenoma, not a fibroma, with a massive amount of ascites and a right-sided hydrothorax. Hence, this case can be categorized as pseudo-Meigs' syndrome.

In gynecological diseases, Meigs' syndrome is rare, with $10-15 \%$ of fibromas presenting with ascites and only $1 \%$ presenting with both ascites and pleural effusions [3]. The reason why this rare syndrome has been highlighted is that this syndrome can be confused with a malignant ovarian neoplasm, carcinomatosis peritoneii or a malignant pleural effusion. Therefore, it is difficult to make an accurate diagnosis before surgery [4].

The pathophysiology of the pleural and peritoneal effusion in Meigs' and pseudo-Meigs' syndrome is unclear but several theories have been proposed. The mechanism of ascites production is normally associated with edematous fibromas or other benign ovarian solid tumors in excess of $10 \mathrm{~cm}$, and the ascites are produced by the lymph released from the surface of the tumor [5]. In particular, a large tumor with a narrow stalk can produce more lymph through intermittent torsion. Moreover, it has been suggested that the presence of fluid results from cyst formation within the tumor as a result of injury or necrosis [3]. The ascites are produced rapidly even before the released ascites are absorbed into the peritoneal membrane.

In the mechanism of the hydrothorax production, ascites are believed to migrate to the right thoracic cavity through a congenital defect or overloaded lymphatics mainly presented in the right-sided diaphragm [6]. Hence, there is a high incidence of the right-sided hydrothorax. This would also explain the disappearance of the hydrothorax after excising the tumor. In some reported cases, the amount of pleural effusion was greater 
than that of ascites, and massive ascites in ovarian carcinomas are not always associated with pleural effusion. Therefore, there might be a factor controlling the accumulation of the fluid released from a benign ovarian tumor [7].

Recently, it was suggested that vascular endothelial growth factor (VEGF), fibroblast growth factor (FGF), and inflammatory cytokine Interleukin- 6 are related to the production of the ascites and the hydrothorax [8]. All 3 factors possess potent vascular permeability-enhancing properties, and have been associated with capillary leakage and the formation of ascites and pleural effusion in other gynecologic abnormalities, such as ovarian hyperstimulation syndrome and ovarian cancer. In particular, the concentrations of these factors are significantly higher in the ascites than in the serum, which suggests that the growth factors mediating the hyperpermeability in the ovary or the peritoneal vessel are released locally rather than systemically.

Patients with Meigs' and pseudo-Meigs' syndromes complain of abdominal distension, abdominal pain, and respiratory distress caused by a massive tumor in the peritoneal cavity and massive ascites and hydrothorax [3]. The increased intraperitoneal pressure caused by the contents in the peritoneal cavity can have adverse physiologic effects, such as decreased cardiac output, ventilation-perfusion abnormalities with an accompanying hypoxia and hypercapnia, reduction in renal perfusion flow and glomerular filtration with decreased urine output, elevation in intracranial pressure, and impaired liver and gastrointestinal perfusion with bowel ischemia and edema. In addition to pleural effusion, the respiratory distress in Meigs' and pseudo-Meigs' syndrome can also be caused by abdominal hypertension due to a progressive increase in the volume of the pelvic mass and ascites.

In this case, there was no respiratory distress before surgery, even though there was a $25 \times 18 \times 8 \mathrm{~cm}$ sized tumor, massive amounts of ascites, and small amounts of a right-sided hydrothorax on the chest radiograph obtained one week before surgery. However, the $\mathrm{PaO}_{2}$ was low in proportion to the $\mathrm{FiO}_{2}$ at the beginning of the incision, and hypoxia occurred during the anesthesia emergence phase along with a remarkably decreased right lung sound. After surgery, the chest radiograph showed total haziness on the right hemothorax, and $750 \mathrm{ml}$ of pleural fluid was drained. It is believed that massive amounts of the hydrothorax caused the decreased mobility of the diaphragm, atelectasis, and decreased lung volume, which further affected the respiratory dysfunction during anesthesia emergence. When a rapid increase in the hydrothorax occurs on the right side, it is possible that the increased amounts of hydrothorax compared to the amounts measured one week earlier can cause a decrease in $\mathrm{PaO}_{2}$ in proportion to $\mathrm{FiO}_{2}$ on the ABGA at the beginning of the incision. However, the increased
$\mathrm{FiO}_{2}$ and applied PEEP helped maintain a $\mathrm{SpO}_{2}$ as $100 \%$.

In other cases, it was also reported that a hydrothorax occurred after gynecologic laparoscopical surgery $[9,10]$. A massive hydrothorax was produced during surgery, particularly at the right-sided chest. After laparoscopic surgery, the $\mathrm{SpO}_{2}$ and $\mathrm{PaO}_{2}$ decreased suddenly, and a chest radiograph revealed a massive right-sided pleural effusion with a displacement of the mediastinum to the left. Large amounts of the hydrothorax were drained by the insertion of a chest tube. It was assumed that the most likely cause of the patient's hydrothorax was irrigation fluid moving from the peritoneal cavity to the pleural space through defects in the diaphragm. In addition, as the hydrothorax related to massive ascites and peritoneal dialysis was reported [11], porous diagram syndrome defining the presence of passage between the abdominal and thoracic cavities was reported [12]. In this case, there was small amount of hydrothorax based on the radiograph one week before surgery. Therefore, it is possible that massive ascites flowed into the thoracic cavity through the diaphragm defects.

Ascites and the hydrothorax can be reproduced rapidly in a short time even before the ovarian tumor is removed surgically. In a case of Meigs' syndrome, it was reported that $600-800 \mathrm{ml}$ of pleural effusion was drained daily via thoracentesis, indicating rapid production of leaking fluid [7]. This increased hydrothorax can cause respiratory distress in any perioperative period.

In conclusion, ovarian tumor, ascites and rapidly increased unilateral pleural effusion strongly suggest the development of pseudo-Meigs' syndrome during the emergence phase in this patient. Serial chest radiography may allow early detection of a significant change in the amount of pleural effusion in patients with an ovarian tumor combined with ascites or pleural effusion. Therefore, although the patient might not have respiratory distress prior to surgery, follow-up chest radiography is recommended. In addition, it is important to focus on respiratory complications that can occur perioperatively, such as respiratory distress and hypoxia.

\section{References}

1. Meigs JV. Pelvic tumors other than fibromas of the ovary with ascites and hydrothorax. Obstet Gynecol 1954; 3: 471-86.

2. Meigs JV. Fibroma of the ovary with ascites and hydrothorax: Meigs' syndrome. Am J Obstet Gynecol 1954; 67: 962-85.

3. Abad A, Cazorla E, Ruiz F, Aznar I, Asins E, Llixiona J. Meigs' syndrome with elevated CA125: case report and review of the literature. Eur J Obstet Gynecol Reprod Biol 1999; 82: 97-9.

4. O' Flanaqan SJ, Tiqhe BF, Eqan TJ, Delaney PV. Meigs' syndrome and pseudo-Meigs' syndrome. J R Soc Med 1987; 80: 252-3.

5. Samanth KK, Black WC III. Benign ovarian stromal tumors associated with free peritoneal fluid. Am J Obstet Gynecol 1970; 107: 538-45. 
6. Chen A, Ho YS, Tu YC, Tanq HS, Chenq TC. Diaphragmatic defect as a cause of massive hydrothorax in cirrhosis of liver. J Clin Gastroenterol 1988; 10: 663-6.

7. Kurai M, Shiozawa T, Noguchi H, Konishi I. Leiomyoma of the ovary presenting with Meigs' syndrome. J Obstet Gynaecol Res 2005; 31: 257-62.

8. Abramov Y, Anteby SO, Fasouliotis SJ, Barak V. Markedly elevated levels of vascular endothelial growth factor, fibroblast growth factor, and interleukin 6 in Meigs' syndrome. Am J Obstet Gynecol 2001; 184: 354-5.

9. Kanno T, Yoshikawa D, Tomioka A, Kamijyo T, Yamada K, Goyo
F. Hydrothorax: an unexpected complication after laparoscopic myomectomy. Br J Anaesth 2001; 87: 507-9.

10. Fukushima A, Okutani R. Unexpected hydrothorax occurring after a long gynecological laparoscopic surgery-a case report. Masui 1997; 46: 413-5.

11. Bergman JM. Complications of peritoneal dialysis related to increased intraabdominal pressure. Kidney Int Suppl 1993; 40: S7580.

12. Kirschner PA. Porous diaphragm syndromes. Chest Surg Clin N Am 1998; 8: 449-72. 